\title{
Rapid processing of low-cost, high-efficiency silicon solar cells
}

\author{
A ROHATGI*, P DOSHI, A EBONG, S NARASIMHA, T KRYGOWSKI and J MOSCHNER ${ }^{\dagger}$ \\ University Centre of Excellence for Photovoltaics Research and Education, School of Electrical and Computer \\ Engineering, Georgia Institute of Technology, 777 Atlantic Dr., Atlanta, GA 30332-0250, USA \\ †Institut für Solarenergieforschung Hamlen/Emmerthal (ISFH), D-31860 Emmerthal, Germany
}

\begin{abstract}
Rapid and potentially low-cost processing techniques are analyzed and applied toward the fabrication of high-efficiency Si solar cells. (i) A technology that can simultaneously form the phosphorus emitter, boron BSF, and in situ oxide in a single high-temperature furnace step or: simultaneously diffused, textured, and AR coated process (STAR) is presented. (ii) A high quality screen-printed (SP) contact methodology is developed that results in fill factors of $0.785-0.790$ on monocrystalline Si. (iii) Aluminum back surface field (Al-BSF) formation is studied in detail to establish the process conditions that result in optimal BSF action. (iv) Screen-printing of Al conductor paste and rapid thermal processing (RTP) are integrated into the BSF procedure, and effective recombination velocities $\left(S_{\text {eff }}\right)$ as low as $200 \mathrm{~cm} / \mathrm{s}$ are demonstrated on $2.3 \Omega-\mathrm{cm}$ Si with this rapid thermal processing of screen-printed contacts, Al alloyed BSF processes. (v) A novel passivation scheme consisting of a dielectric stack (plasma silicon nitride on top of a rapid thermal oxide) is developed to reduce the surface recombination velocity $(S)$ to $\approx 10 \mathrm{~cm} / \mathrm{s} \cdot$ at the $1.3 \Omega-c m$ Si surface. The important feature of this stack passivation scheme is its ability to withstand a high-temperature anneal $\left(700-850^{\circ} \mathrm{C}\right)$ without degradation in surface recombination velocity. This feature is critical for most current commercial processes that utilize SP contact firing. (vi) Finally, the individual processes are integrated to form high-efficiency, manufacturable devices. Solar cell efficiencies of $17 \%$ and $>19 \%$ are achieved on FZ Si with SP and evaporated (photolithography) contacts, respectively.
\end{abstract}

Keywords. RTP; silicon; surface passivation; recombination; screen printing; back surface field; fill factor; solar cell.

\section{Introduction}

For widespread implementation of silicon photovoltaics (PV), the module cost must be reduced by a factor of 2 to 4 . This can be accomplished by lowering the cost of solar cell materials and processing without sacrificing cell efficiency. In an attempt to reduce manufacturing cost, most current manufacturers use a minimal number of low-cost processing steps. The resulting cell efficiencies are in the 10-15\% range. In contrast to industrial cells, laboratory cells have reached efficiencies over $24 \%$ (Zhao et al 1995). However, expensive processing techniques and numerous processing steps have been used to incorporate high efficiency features such as selective emitter, light trapping, low front and back surface recombination velocity, and contacts formed by evaporation and photolithography. Unfortunately, current laboratory cells are too expensive and industrial cells are not efficient enough to meet the cost and efficiency targets simultaneously.

In addition, to reach $50-100 \mathrm{MW} /$ year production capacity, a PV manufacturing plant must produce less than one cell per second (Mitchell et al 1994). Processes must be invented that can significantly reduce the processing time without sacrificing efficiency, and corres-

*Author for correspondence pondingly equipment must be developed that can handle more wafers per unit time. Therefore, the objective of this paper is to develop processes that are not only fast but also capable of yielding high efficiency. This study presents rapid and improved formation of screen-printed (SP) contacts, Al alloyed BSFs, front and back surface passivation, $n^{+}$emitter regions, and the integration of all these technologies to achieve high efficiency cells.

\section{Simultaneously diffused, textured and $\mathbf{A R}$} coated (STAR) process

The STAR process is being developed at Georgia Tech, which incorporates a simultaneously diffused emitter and back surface field (BSF) on a textured silicon wafer, with an in situ thermal oxide for surface passivation and anti-reflection coating. In a single high temperature step, the STAR process provides five important efficiency enhancement features: (i) front and back doping profiles compatible with high efficiency cell design, (ii) emitter oxide passivation, (iii) back surface passivation via a passivated boron back surface field, (iv) a low reflectance single layer $\mathrm{SiO}_{2}$ AR coating on the textured surface, and (v) $\mathrm{Al}-\mathrm{SiO}_{2}-\mathrm{Si}$ back surface reflector for light trapping. The STAR process involves in-house fabrication of doping sources using silicon wafers coated with $P$ and 
B compounds. The phosphorus sources are prepared by a $30 \mathrm{~min} \mathrm{POCl}_{3}$ diffusion or simply by a spin-on process followed by a short bake. Boron sources are formed by a liquid spin-on process. By controlling the concentration of $\mathrm{P}_{2} \mathrm{O}_{5}$ and $\mathrm{B}_{2} \mathrm{O}_{3}$ on the same wafers, a wide range of emitter and BSF profiles can be obtained simultaneously. The resulting $P$ diffusion glass on the solar cell wafer is only $50 \AA$, which allows in situ growth of high quality oxide without appreciably increasing the reflectance of the device. It is found that the use of separate source wafers not only gives more flexibility in controlling front and back sheet resistances for the same thermal cycle, but also acts as a powerful contamination filter, especially in the case of boron. After each simultaneous diffusion cycle, the source wafers are recycled by a brief dip in $10 \% \mathrm{HF}$ followed by the short spin-on process.

Figure 1 shows the source-sample arrangement in the STAR process which is performed in a conventional diffusion furnace. After surface texturing, $\mathrm{P}$ and $\mathrm{B}$ diffusions are performed simultaneously at $1000 \mathrm{C} / 60 \mathrm{~min}$ followed by a $60 \mathrm{~min}$ in situ oxidation, resulting in 90 $\Omega / \square$ sheet resistance on the front, $30 \Omega / \square$ on the back, and $\sim 1100 \AA$ front and back oxide for surface passivation. Front oxide also serves as a single layer AR coating, while back oxide forms a back surface reflector. This is followed by two photomask steps: one for the front grid and the other for point contacts to the $p^{+}$region. This process has so far produced $\sim 20 \%$ efficient STAR I cells (figure 2). However, if the boron is spun-on directly onto the sample wafer, the bulk lifetime drops to $6 \mu \mathrm{s}$ from $1 \mathrm{~ms}$, resulting in a cell efficiency of only $15 \%$. This supports the merit of using separate source wafers to filter contaminants in the spin-on solution. Detailed modelling and analysis of these $20 \%$ cells showed that further improvements are possible by incorporating double layer AR coating, a flat back surface, and making the boron BSF more transparent by increasing its sheet resistance and lowering diffusion temperature (STAR II cell).

In the next generation STAR cells (STAR III), photolithography will be replaced by screen-printed contacts. It is also found that the sheet resistance in the textured regions is twice that of the flat regions in the STAR

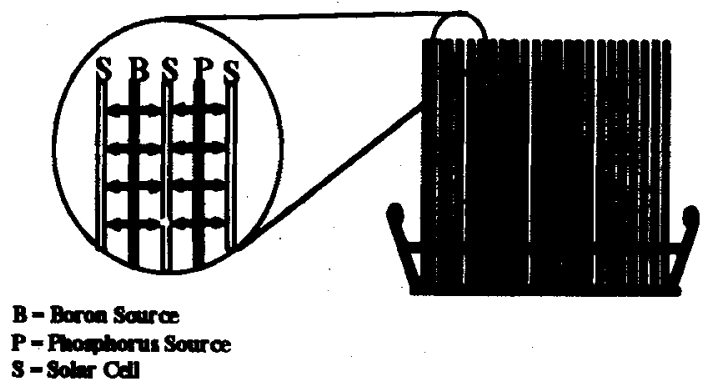

Figure 1. Source/sample arrangement for STAR process. process, which uses limited sources. This will help in the formation of selective emitters without double diffusion, provided a texturing mask can be screen-printed in the grid regions. In addition, local BSF will be formed by screen printing $\mathrm{Al}$ through the passivating back oxide. Thus, next generation STAR cells will only use one furnace step and three screen-printing steps. Preliminary model calculations show that greater than $18 \%$ efficient cells are possible on a silicon material with a lifetime of about only $50 \mu \mathrm{s}$. STAR II and III cells are currently in the development stage.

\section{A methodology for achieving high-quality screen-printed contacts}

One of the most difficult aspects of large-scale solar cell production is forming low-cost, high-quality front contacts. Screen-printing (SP) offers a simple, costeffective contact method that is consistent with the requirements for high-volume manufacturing. The current problem with SP, however, is that the throughput gains are attained at the expense of device performance.

The primary fill factor loss mechanisms associated with SP metallization are: (i) gridline resistance, (ii) contact resistance, and (iii) junction leakage and shunting. The gridline resistivity and the contact resistance both depend on the contact firing cycle and the material quality of the conductor paste. The junction leakage and shunting behaviour depend primarily on the junction depth and the contact firing cycle. In the following sections, a methodology for optimization of SP metallization in the context of these parameters is presented.

\subsection{Effect of SP firing treatment on conductor paste resistivity}

The conductor paste used in this work was made by Ferro Corporation (3349 Ag Conductor). After printing, the following procedure was used to form the contacts.

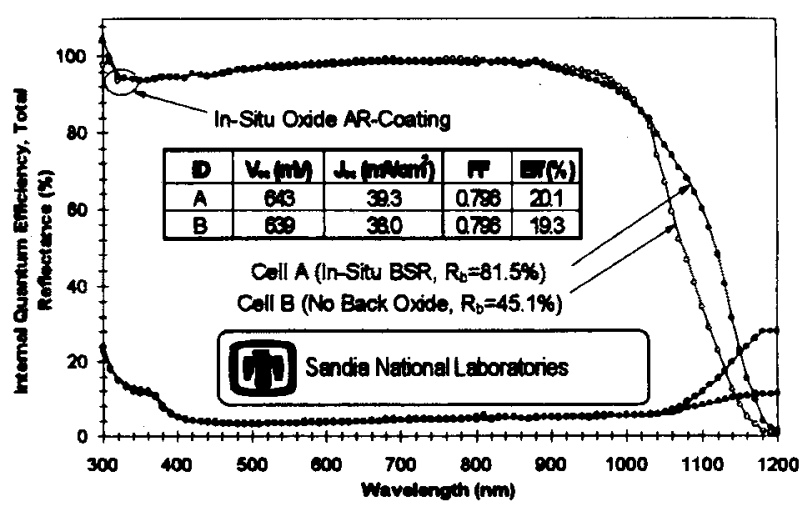

Figure 2. IQE for $20 \%$-efficient STAR cells. 
First, the solvents were removed by baking on a hotplate at $150^{\circ} \mathrm{C}$ for $2 \mathrm{~min}$. This was followed by firing in a 3-zone IR-belt furnace in which the lengths of zones 1 , 2 , and 3 were $7.5,15$, and 7.5 inches, respectively. The first two zones were set to $425^{\circ} \mathrm{C}$ and $580^{\circ} \mathrm{C}$ and used to burn off organic materials in the printed paste. The hotzone (zone 3 ) temperature was varied to suit the particular investigation. The overall firing time was determined by the beltspeed through the furnace. Beltspeeds of 15 and 40 inches/min were implemented in this study, which correspond to hotzone dwell times of 30 and $11 \mathrm{sec}$, respectively.

As shown in figure $3, \rho_{\text {metal }}$ is a strong function of hotzone firing temperature. For a dwell time of $30 \mathrm{sec}$ (beltspeed of $15^{\prime \prime} / \mathrm{min}$ ), $\rho_{\text {metal }}$ decreases by a factor of $\approx 2$ (from 5.3 to $2 \cdot 2 \mu \Omega-\mathrm{cm}$ ) by increasing the hotzone temperature from $550^{\circ} \mathrm{C}$ to $850^{\circ} \mathrm{C}$. (It is instructive to compare these $\rho_{\text {metal }}$ values to the resistivity of pure $\mathrm{Ag}$, $1.6 \mu \Omega-\mathrm{cm}$.) At higher beltspeed, the overall process time is reduced. To compensate for this effect, the temperature setpoints must be increased. Figure 3 shows that the $\rho_{\text {metal }}$ of $\approx 3.3 \mu \Omega$-cm (compatible with fill factors of 0.780 for $4 \mathrm{~cm}^{2}$ cells) can be achieved by either $700^{\circ} \mathrm{C}$ firing at a beltspeed of $37.5 \mathrm{~cm} / \mathrm{min}$ or $850^{\circ} \mathrm{C}$ firing at a beltspeed of $100 \mathrm{~cm} / \mathrm{min}$.

\subsection{Optimization of emitter junction depth for reducing leakage and shunt effects}

Several phosphorus-diffused emitters were formed resulting in $n^{+}$sheet resistance between 40 and $90 \Omega / \mathrm{sq}$ and junction depth between 0.25 and $0.50 \mu \mathrm{m}$ (figure 4). SP contacts were fired at a hotzone temperature of $730^{\circ} \mathrm{C}$ in $30 \mathrm{sec}$. The resulting fill factors are shown in figure

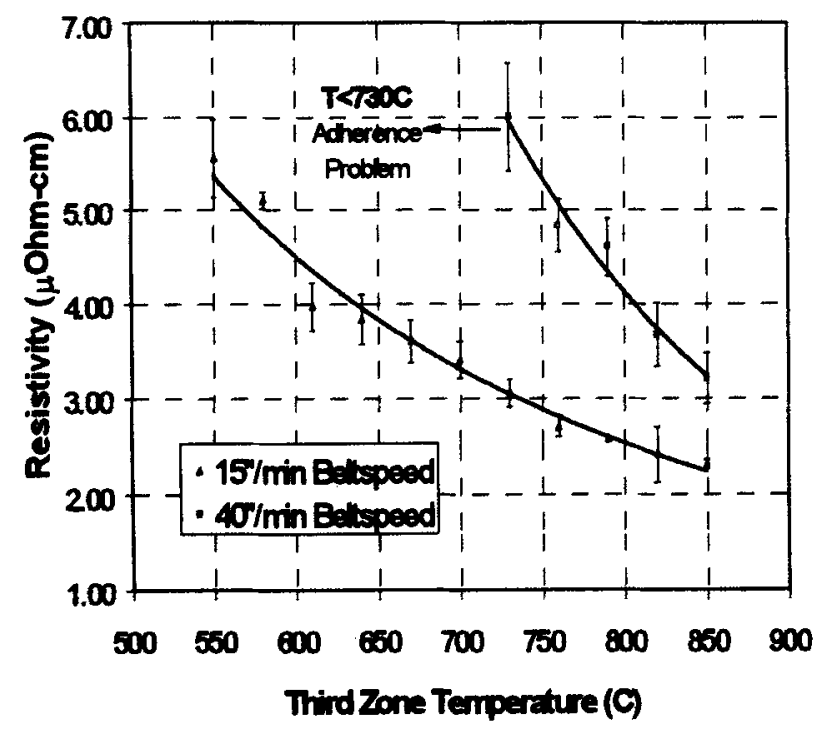

Figure 3. Effect of hotzone firing temperature and beltspeed on metal conductivity. The hotzone dwell times were $30 \mathrm{sec}$ $(37.5 \mathrm{~cm} / \mathrm{min})$ and $11 \mathrm{sec}(100 \mathrm{~cm} / \mathrm{min})$.
5 as a function of $n^{+}$sheet resistance and emitter junction depth $\left(x_{\mathrm{j}}\right)$. It is clear that the fill factor improves with junction depth. Dark IV measurements of these devices revealed that junction shunting and leakage are the primary loss mechanisms when $x_{\mathrm{j}}$ goes below $0.4 \mu \mathrm{m}$. When $x_{\mathrm{j}}$ is increased to $\approx 0.5 \mu \mathrm{m}$, negligible leakage current $\left(J_{02}<10^{-8} \mathrm{~A} / \mathrm{cm}^{2}\right)$ and high shunt resistance $\left(R_{\mathrm{sh}}>10^{3} \Omega-\mathrm{cm}^{2}\right)$ are measured, and solar cells with fill factors of $0.785-0.790$ are achieved.

\subsection{Effect of post-firing forming gas anneal on the contact resistance and fill factor}

In addition to $R_{\mathrm{sh}}$ and $J_{\mathrm{o} 2}$, the quality of SP contacts depends on the $R_{\text {series. }}$. The gridline resistance component of $R_{\text {series }}$ was discussed above. In this section, the contact resistance $\left(\rho_{\mathrm{v}}\right)$ associated with SP metallization is examined. A low-temperature FGA is found to be extremely effective in lowering $\rho_{\mathrm{c}}$ after the SP contacts have been fired in the IR belt furnace.

The effect of hotzone firing condition on fill factor was investigated for large temperature variations. The response is shown in figure 6 for beltspeeds of $37.5 \mathrm{~cm} / \mathrm{min}$ and $100 \mathrm{~cm} / \mathrm{min}$. Immediately after contact firing, the fill factors are prohibitively low $(\approx 0.500-0.600)$. These fill factors improve drastically when the samples are annealed in forming gas at $400^{\circ} \mathrm{C}$. This process reveals that there exists at least a $60^{\circ} \mathrm{C}$ range in acceptable hotzone firing temperature that results in fill factors of 0.785 0.790 . For the $100 \mathrm{~cm} / \mathrm{min}$ beltspeed, a similar range exists, though higher process temperatures are required to offset the reduced dwell time and increased ramp-up distance.

Measurements of $\rho_{\mathrm{c}}$ by the transmission line model (figure 7) provide clear evidence of $\rho_{\mathrm{c}}$ reduction due to FGA treatment. The final $\rho_{\mathrm{c}}$ is observed to be rather invariant to $n^{+}$sheet resistance.

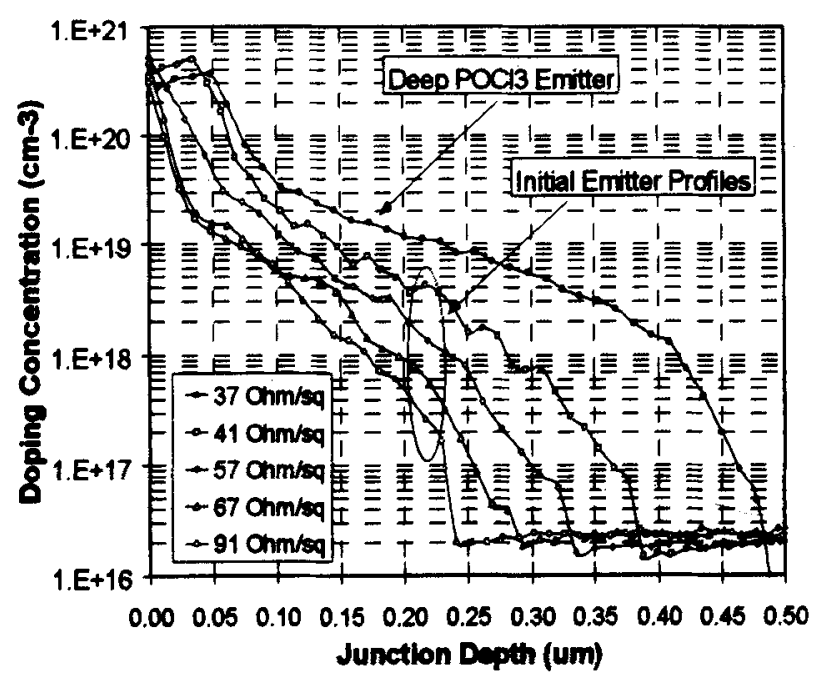

Figure 4. Emitter structures used for fill factor investigation. 


\section{Rapid phosphorus diffusion by spin-on dopants and RTP}

Phosphorous diffusion in a conventional furnace takes more than one hour, which could limit the throughput of a manufacturing line. This section describes the investigation of emitter formation in an RTP system that uses tungsten halogen lamps to optically heat the sample. At the diffusion temperature, these lamps produce highenergy photons that increase the effective diffusion of phosphorous into silicon when used in conjunction with spin-on dopants (SOD). Figure 8 shows that, after SOD application, an $880^{\circ} \mathrm{C} / 10 \mathrm{~min}$ RTP drive-in produces significantly more diffusion compared to a conventional furnace (primarily infrared photon excitation). Schindler (1998) has recently proposed that this is the result of high energy photon-induced modification of the SOD or SOD/Si interface rather than an enhanced diffusivity of phosphorous in $\mathrm{Si}$. We have been able to achieve 40-80 $\Omega / \mathrm{sq}$ RTP emitters in less than $5 \mathrm{~min}$ using a SOD, and integrate them into cells without sacrificing any efficiency performance. These devices are discussed in $\S 7$.

\section{Development of a rapid and improved aluminum back surface field for silicon solar cells}

The Al-BSF is attractive for reducing $S_{\text {eff }}$ in $\mathrm{Si}$ solar cells because the $p^{+}$region can be formed by a quick alloying step instead of a lengthy diffusion procedure. However, the electrical quality of an Al-BSF is extremely sensitive to process conditions. If the $p^{+}$is not formed appropriately, full performance gains will not be realized. In this section, the Al-Si system is studied from a fundamental standpoint to establish BSF formation conditions that result in the lowest $S_{\text {eff }}$ response.

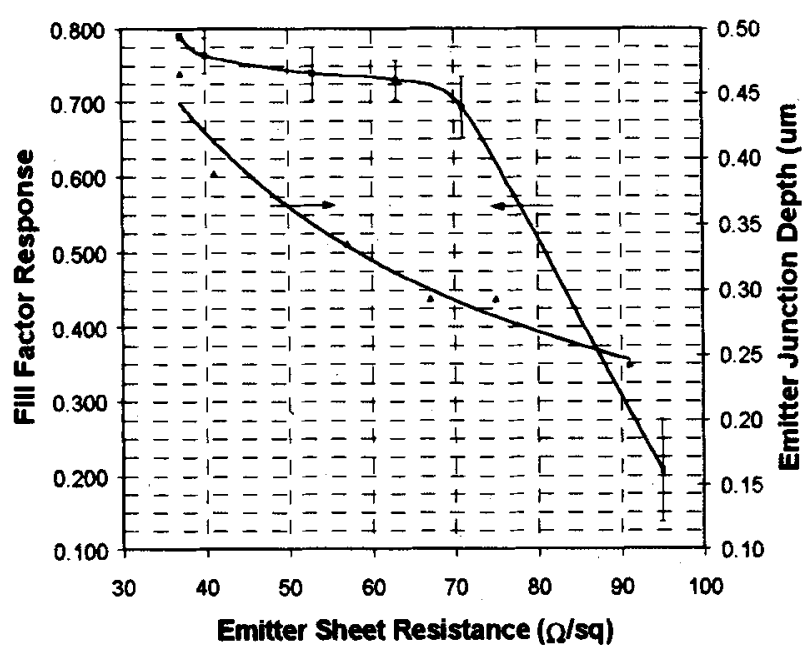

Figure 5. Effect of $x_{\mathrm{j}}$ and $\rho_{\text {sheet }}$ on fill factor response.
The uniformity of an Al-BSF is controlled to a large extent by the ramp-up rate used to reach the alloying temperature (Roberts and Wilkinson 1968; Chalfoun 1996). Under slow ramp conditions, alloying between $\mathrm{Al}$ and $\mathrm{Si}$ can occur at certain sites before others (a form of local wetting) resulting in a nonuniform Al-BSF. These nonuniformities can include variations in junction depth, loss of surface planarity, Al spiking, and possibly nonformation of the $p^{+}$region. Under fast ramp conditions, a sample goes through the eutectic point and reaches the process temperature very quickly. At typical process temperatures $\left(\approx 800-900^{\circ} \mathrm{C}\right)$, the $\mathrm{Al}$ layer becomes molten and readily wets the entire $\mathrm{Si}$ surface. This promotes more uniform alloying, which in turn improves Al-BSF uniformity. The purpose of this study is to determine the impact of this effect on $S_{\text {eff }}$ and solar cell performance.

In order to correlate the degree of BSF uniformity to device performance, two samples of BSFs were formed by thermally evaporating $10 \mu \mathrm{m}$ of $\mathrm{Al}$ onto the $\mathrm{Si}$ substrates and then alloying at $850^{\circ} \mathrm{C}$. The sample undergoing the slow ramp process was pushed into a conventional furnace below the $\mathrm{Al}-\mathrm{Si}$ eutectic temperature and ramped-up at a rate of $5 \% \mathrm{~min}$. The sample undergoing the fast ramp procedure was alloyed in a rapid thermal processing (RTP) unit and ramped-up at a rate of $1200^{\circ} / \mathrm{min}$. After processing, the $p^{+}$regions were delineated by etching in an acid solution (Runyan 1975). Figures $9 \mathrm{a}$ and $\mathrm{b}$ show cross sectional SEM micrographs of the Al-BSF regions formed under slow ramp and fast ramp conditions. Clearly, the slow ramp process results in significant nonuniformities in the $p^{+}$junction (pitting and non-formation across much of the wafer surface) (figure 9a). On the contrary, the sample alloyed under fast ramp conditions shows a higher degree of junction uniformity and planarity (figure 9b). This effect was first discussed by Roberts and Wilkinson (1968), and additional evidence was presented Chalfoun (1996).

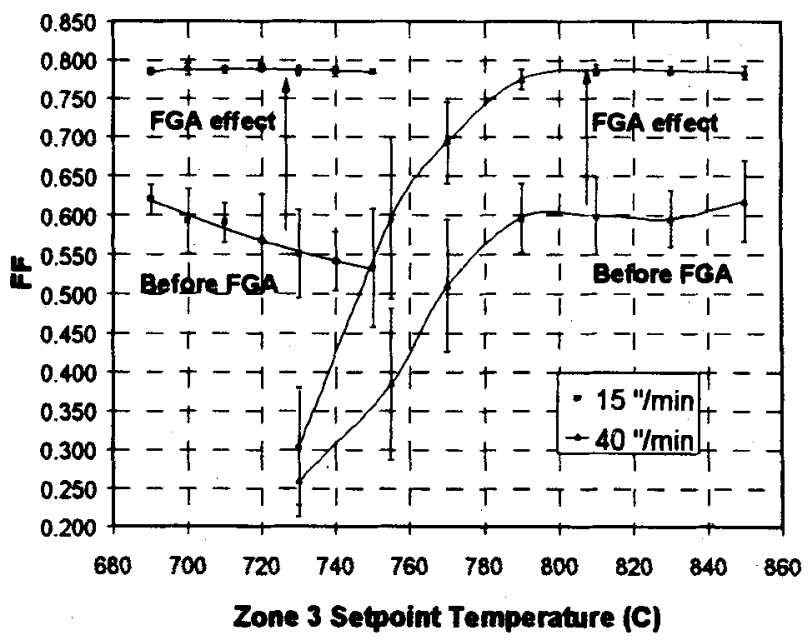

Figure 6. Fill factor response as a function of hotzone temperature and dwell time. 
IQE measurements in figure 10 show that the BSF uniformity variation has a significant impact on the performance of solar cells formed on materials in which the ratio of minority carrier diffusion length $\left(L_{\mathrm{d}}\right)$ to cell width $(W)$ exceeds unity. Comparison of the measured and simulated IQE data in figure 10 shows that $S_{\text {eff }}$ is reduced by nearly one order of magnitude, from $1000 \mathrm{~cm} / \mathrm{s}$ to $100-200 \mathrm{~cm} / \mathrm{s}$, by changing from the slow ramp to fast ramp alloying condition.

Al-BSF modelling predicts that increasing the Al deposition thickness should have a strong effect on the resulting $S_{\text {eff }}$ (Narasimha et al 1998). Figure 11 shows that this theoretical prediction is most accurate only when fast-ramp alloying is implemented. Under such conditions, $V_{o c}$ improvements in excess of $25 \mathrm{mV}$ can be achieved by increasing the $\mathrm{Al}$ deposition quantity from $1 \mu \mathrm{m}$ to $10 \mu \mathrm{m}$ for $2.3 \Omega-\mathrm{cm} \mathrm{FZ} \mathrm{Si} \mathrm{substrate}$ material.

Thick film Al deposition can readily be achieved by screen-printing Al conductor paste onto the Si substrate. Figure 11 also indicates that optimal results are obtained when a thick SP Al film is RTP alloyed in 2 min at $850^{\circ} \mathrm{C}$. (Higher alloying temperatures were observed to inject impurities from the paste into the bulk, thereby reducing carrier lifetime and cell performance.)

\section{Development of a novel RTO/SiN stack for effective front and back surface passivation of silicon solar cells}

As shown above, the SP Al-BSF can be extremely effective in reducing $S_{\mathrm{b}}$ in $\mathrm{Si}$ solar cells. However, a concern with the Al-BSF process is that the stresses imparted to the substrate during formation could preclude application to thin wafers. Process-induced stress or wafer warping can be virtually eliminated by employing a passivated rear structure in which the rear side metalli-

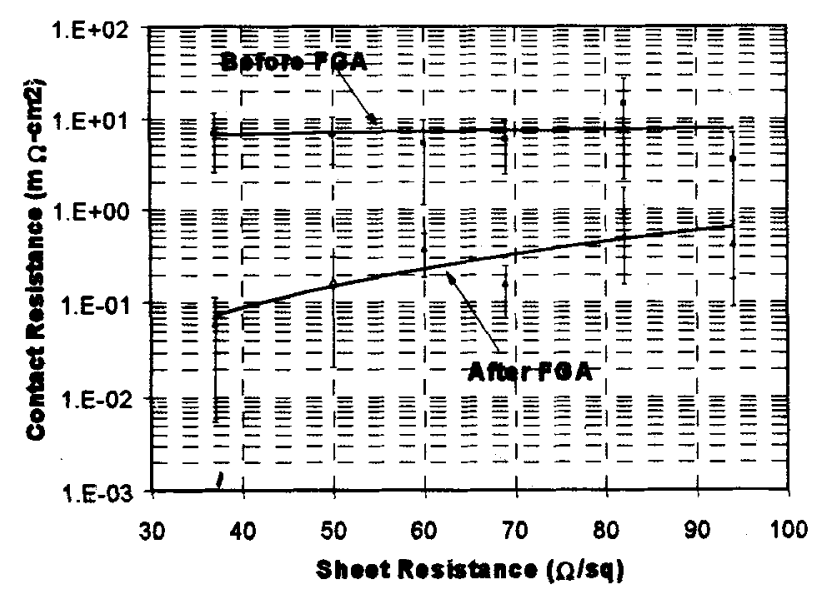

Figure 7. The effect of the FGA on the $\rho_{c}$ of SP contacts to $n^{+}$emitter regions. zation covers only a small fraction of the surface area. In addition to reducing process-induced stress, this structure is well suited for bifacial operation since the rear surface is transparent to incoming light. However, in order to take advantage of this structural feature and attain a significant power output, the passivation at the rear surface must be highly effective.

In this $\S$, a novel dielectric stack comprised of a rapid thermal oxide (RTO) and a direct plasma silicon nitride ( $\mathrm{SiN})$ is developed for effective passivation of the low resistivity $(0.65-1.3 \Omega-\mathrm{cm})$ p-type (100) $\mathrm{Si}$ surface. The essential feature of the stack passivation scheme is its ability to withstand moderate temperature annealing $\left(700-850^{\circ} \mathrm{C}\right)$ without any degradation in surface recombination velocity. In fact, the stack relies on this type of thermal treatment to achieve low $S$ values. The effectiveness of three surface passivation techniques (RTO alone, $\mathrm{SiN}$ alone, and the stack passivation) are compared in figure 12.

The RTO layer alone results in $S>10,000 \mathrm{~cm} / \mathrm{s}$ immediately after growth. This extremely poor $S$ can be reduced to $\sim 100 \mathrm{~cm} / \mathrm{s}$ if an additional FGA at $400^{\circ} \mathrm{C}$ is performed. However, a subsequent $730^{\circ} \mathrm{C}$ IR-belt anneal (simulating SP contact firing) degrades the passivation and increases $S$ above $1000 \mathrm{~cm} / \mathrm{s}$. A similar trend is observed in this study for the PECVD SiN film alone. The as-deposited SiN results in $S>10,000 \mathrm{~cm} / \mathrm{s}$ which is reduced to less than $200 \mathrm{~cm} / \mathrm{s}$ by an ensuing anneal in forming gas at $400^{\circ} \mathrm{C}$. The $730^{\circ} \mathrm{C}$ beltline anneal again degrades the interface quality, and increases $S$ by roughly one order of magnitude.

Contrary to the response of these individual films, annealing the RTO/PECVD SiN stack actually enhances the passivation quality. The stepwise effect of stacking PECVD SiN on top of the RTO layer and then annealing at $730^{\circ} \mathrm{C}$ is evident in figure 12 . An $S$ value of nearly $10 \mathrm{~cm} / \mathrm{s}$ is attained after the final anneal (step 3) which

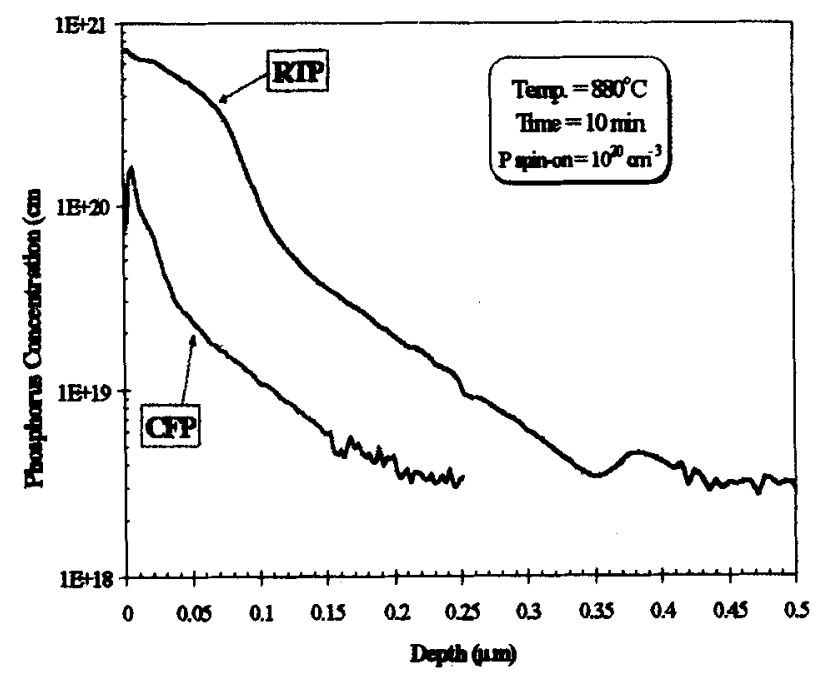

Figure 8. RTP vs CFP diffusion of SOD film. 
is far superior to the $S$ value after RTO growth (step 1) or SiN deposition on top of the RTO (step 2). The $730^{\circ} \mathrm{C}$ anneal is believed to enhance the release and delivery of atomic hydrogen from the $\mathrm{SiN}$ film to the $\mathrm{Si}-\mathrm{SiO}_{2}$ interface, thereby reducing the $D_{\mathrm{it}}(E)$ at the surface.

In addition to passivating the low-resistivity $p$-type $\mathrm{Si}$ surface, the stack is also found to be very effective in lowering $S$ at phosphorus diffused surfaces. This is shown for both $40 \Omega / \mathrm{sq}$ and $90 \Omega / \mathrm{sq}$ emitter regions in figures 13 and 14 , respectively.
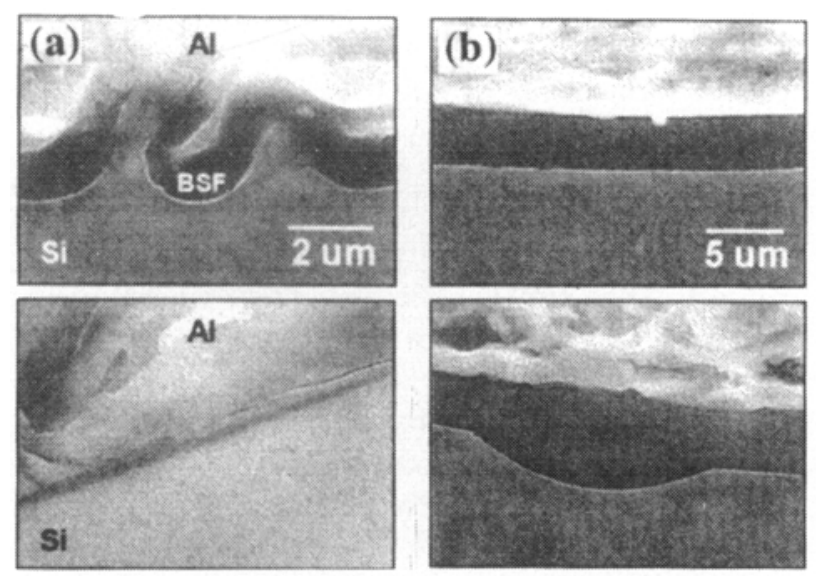

Figure 9. BSF regions formed under (a) slow ramp conditions (top shows severe junction nonuniformity, bottom shows nonformation) and (b) fast ramp conditions (both top and bottom show clean formation and improved uniformity).

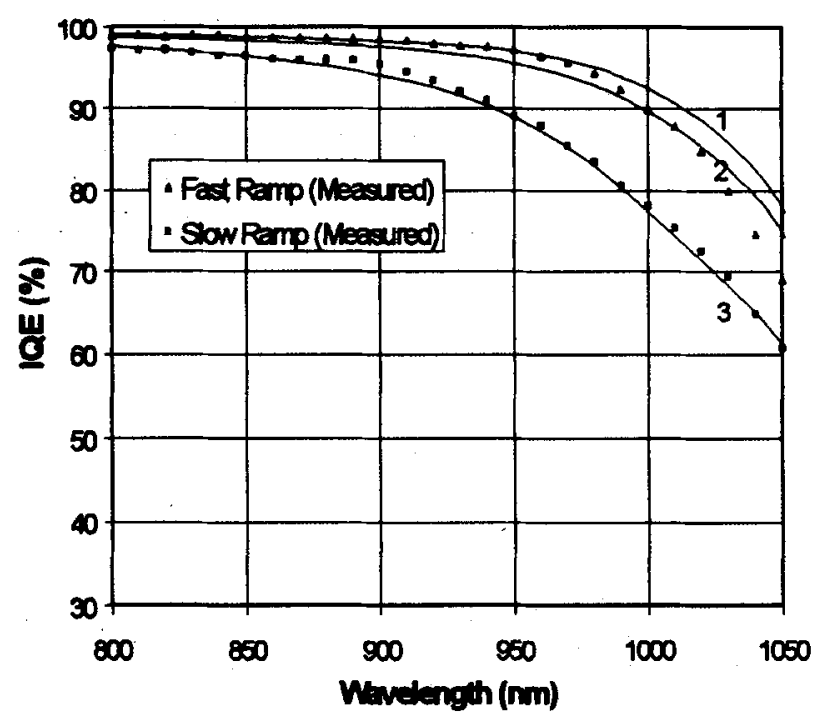

Figure 10. Effect of ramp rate on the long wavelength IQE of Al-BSF solar cells. The solid lines represent simulation results: $1 . S_{\mathrm{b}}=100 \mathrm{~cm} / \mathrm{s}, 2 . S_{\mathrm{b}}=200 \mathrm{~cm} / \mathrm{s}$, and $3 . S_{\mathrm{b}}=1000 \mathrm{~cm} / \mathrm{s}$. The data points were measured from Al-BSF cells with $10 \mathrm{~m}$ evaporated $\mathrm{Al}$ alloyed at $850^{\circ} \mathrm{C}$.
The stack passivation scheme has been used to fabricate fully screen-printed bifacial solar cells. Preliminary studies have resulted in bifacial cells with rear-illuminated efficiencies as high as $11.6 \%$ using this passivation scheme.

\section{Cell fabrication by integration of rapid technologies}

Our simple baseline laboratory process uses conventional furnace processing (CFP) to form an $80 \Omega / \mathrm{sq}$ emitter, front oxide passivation, and $<1 \mu \mathrm{m}$ deep Al BSF. Contacts

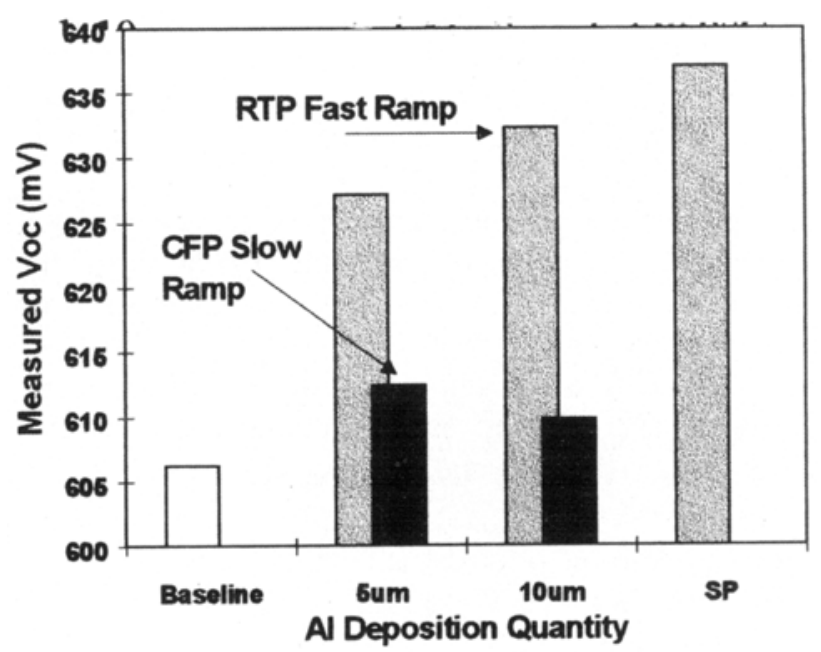

Figure 11. The effect of $\mathrm{Al}$ deposition thickness and ramp-rate on solar cell $V_{\mathrm{oc}}(2.3 \Omega-\mathrm{cm} \mathrm{FZ} \mathrm{Si}$ substrate). The CFP (black bars) and RTP (gray bars) ramp rates were $5 \%$ min and $1200 \% \mathrm{~min}$, respectively, and the peak alloy temperature was $850^{\circ} \mathrm{C}$.

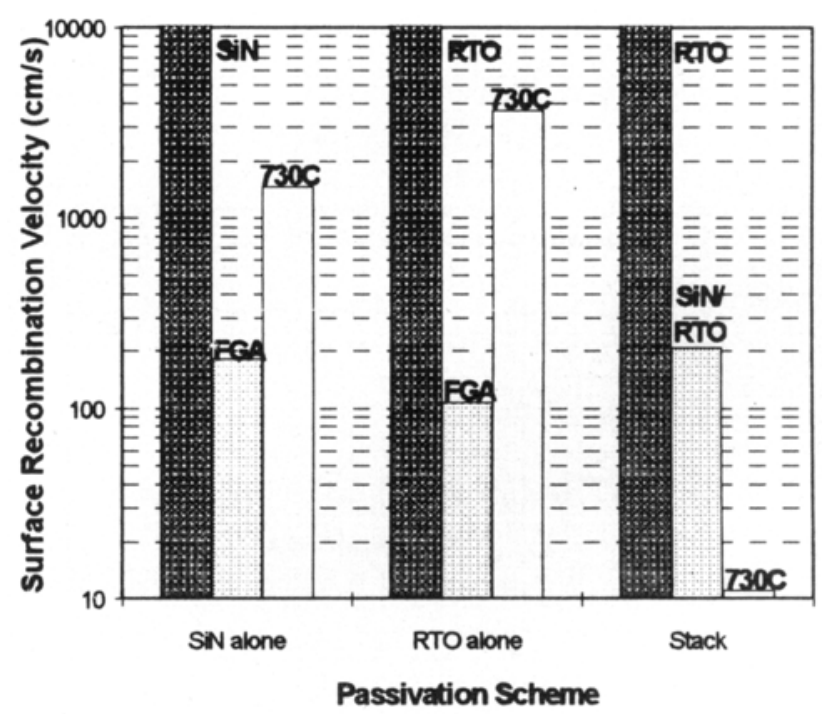

Figure 12. Stack passivation scheme compared with individual RTO and SiN. $S$ values determined by the PCD technique (Schroder 1997). 


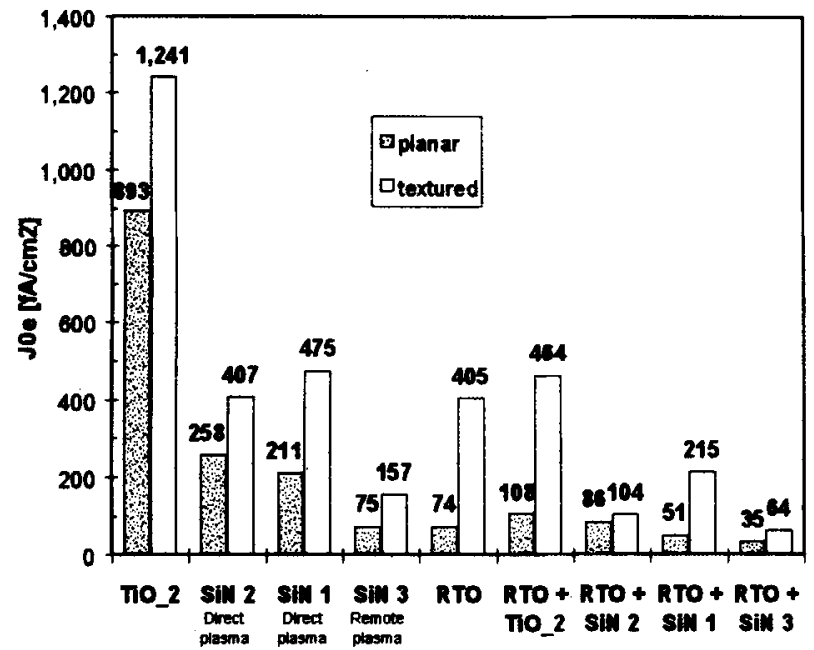

Figure 13. $J_{\text {oe }}$ values for $40 \Omega / \mathrm{sq}$ emitters. SiN 3 represents remote PECVD SiN.

are formed by metal evaporation and photolithography on the front and back. This process involves $6 \mathrm{~h}$ of high temperature processing, $2 \frac{1}{2} \mathrm{~h}$ of metal evaporation, and $5 \mathrm{~h}$ of photolithography; yielding a cell efficiency of $\sim 17.8 \%$ on $1 \Omega-c m$ FZ silicon without any texturization.

The above process was modified by accomplishing the $n^{+}$emitter diffusion, front surface oxide passivation, and SP Al-BSF alloying all within an RTP unit. The $80 \Omega / s q$ emitter was formed in $6 \mathrm{~min}$ by application of a SOD followed by a short bake and an RTP drive-in. Next, Al paste was screen-printed on the back followed by $850^{\circ} \mathrm{C} / 2$ min RTP drive-in in $\mathrm{O}_{2}$. During this same step, a rapid thermal oxide (RTO) was simultaneously grown on the front surface. This RTP process not only reduced the cell processing time from $16 \mathrm{~h}$ to $8 \mathrm{~h}$, but also produced a higher cell efficiency of $19.2 \%$ because of the superior $\mathrm{Al} \mathrm{BSF}$ and equally effective RTO.

In the next phase, the $80 \Omega / s q n^{+}$region was replaced by a $40 \Omega / \mathrm{sq}$ RTP-diffused emitter, and the photolithography front contacts were replaced by SP contacts. This RTP/SP process reduced the cell processing time to $2 \mathrm{~h}$ and gave a cell efficiency of $17 \%$.

One final obstacle impeding widespread application of RTP is the lack of high-throughput RTP machine. Work presented up till now in this paper utilizes a single wafer RTP (SW-RTP) system. Recently, we have been developing a process using continuous beltline rapid thermal processing (BL-RTP). A commercial beltline system equipped with tungsten-halogen lamps has been used to perform $n^{+}$emitter diffusion, BSF formation, and SP contact firing. This process has resulted in $17.0 \%$ efficient cells on $1.3 \Omega-\mathrm{cm} \mathrm{FZ} \mathrm{Si}$.

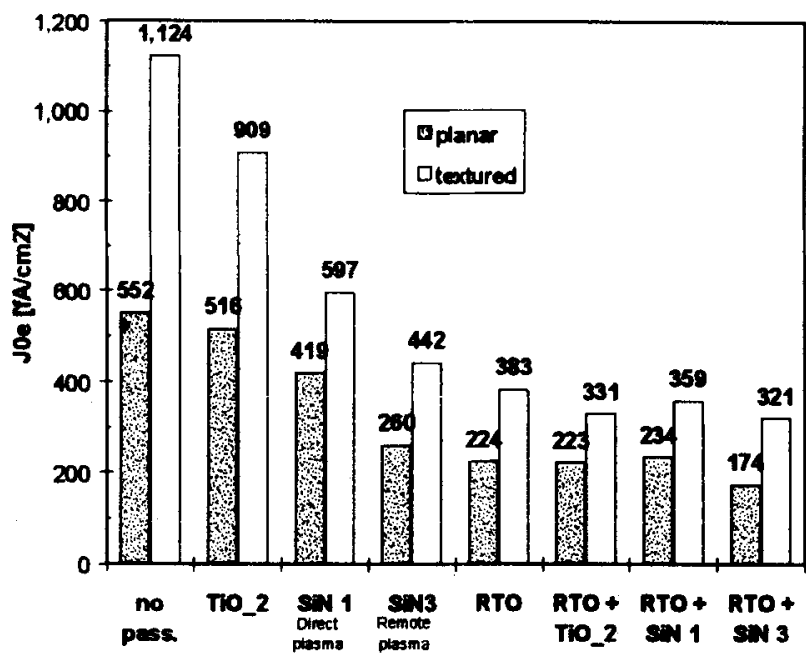

Figure 14. $J_{\text {oe }}$ values for $90 \Omega / \mathrm{sq}$ emitters. SiN 3 represents remote PECVD SiN.

\section{Conclusions}

A technology involving simultaneous emitter and BSF diffusion and in situ oxidation was developed in the STAR process. This process has so far resulted in $20 \%$ efficient cells using evaporations and photolithography for contacts. The next step was to optimize lower-cost SP contacts to result in a more manufacturable process. Thus, a methodology was developed to achieve high quality SP contacts with fill factors in the $0.785-0.790$ range for mono-crystalline $\mathrm{Si}$ cells. It was shown that an RTP alloyed, SP Al-BSF, formed in 2 min yielded a very low $S_{\text {eff }}$ of $200 \Omega-\mathrm{cm} / \mathrm{s}$ on $2.3 \Omega-\mathrm{cm} \mathrm{FZ} \mathrm{Si.} \mathrm{In}$ the same thermal cycle, an effective RTO layer could be grown for front surface passivation. A novel stack passivation scheme (consisting of plasma SiN stacked on top of an RTO layer) was developed which could attain $S$ values approaching $10 \mathrm{~cm} / \mathrm{s}$ on $1.3 \Omega-\mathrm{cm} \mathrm{Si}$, and $J_{\text {oe }}$ values as low as $174 \mathrm{fA} / \mathrm{cm}^{2}$ and $35 \mathrm{fA} / \mathrm{cm}^{2}$ on $40 \Omega / \mathrm{sq}$ and $90 \Omega / \mathrm{sq}$ phosphorous-diffused emitters, respectively.

Using RTP, formation of the $n^{+}$emitter, front surface oxide passivation, and Al-BSF resulted in a substantial reduction in cell processing time. Cell efficiencies of $19.2 \%$ and $17 \%$ have been achieved on $\mathrm{FZ} \mathrm{Si}$ using photolithography and SP contact techniques, respectively. Finally, rapidly formed SP cells in a commercial beltline machine also resulted in $17 \%$ efficiency, endorsing the potential of RTP for cost-effective Si photovoltaics.

\section{Acknowledgements}

This work was supported by Sandia National Labs under Subcontract No. AO-6162 and NREL under Subcontract No. XD-2-11004-2. 


\section{References}

Chalfoun L L 1996 Process optimization of alloyed aluminum back side contact for silicon solar cells, Master Thesis, Massachusetts Institute of Technology, Massachusetts

Mitchell K, King R, Jester T and McGraw M 1994 Conf. record of 24th IEEE photovoltaic specialists conference (Piscataway: IEEE) pp 1266-1269
Narasimha S, Rohatgi A and Weber A 1999 IEEE Trans. Electron Dev. (accepted)

Roberts F M and Wilkinson L G 1968 J. Mater. Sci. 3110

Runyan W R 1975 Semiconductor measurements and instrumentation (New York: McGraw Hill)

Schindler S et al 1998 Appl. Phys. Lett. 722583

Schroder D K 1997 IEEE Trans. Electron. Dev. 44160

Zhao J, Wang A, Altermatt P and Green M A 1995 Appl. Phys. Lett. 663636 